\title{
CONF-950846--37
}

SAND95-1936C

\section{INVESTIGATION OF THIN LASER-DRIVEN FLYER PLATES USING STREAK IMAGING AND STOP MOTION MICROPHOTOGRAPHY*}

\author{
Alan M. Frank \\ Lowrence Livermore National Laboratory, Livermore; CA 94551-9900 \\ and \\ Wayne M. Trott \\ Sandia National Laboratories, Albuquerque, NM 87185-0834
}

\begin{abstract}
The dynamic behavior of laser-accelerated flyers has been studied using high-speed streak imaging in combination with stop motion microphotography. With very thin targets, melting and plasma penetration of the flyer material occur in rapid sequence. The time delay from the onset of motion to flyer breakup increases with flyer thickness and decreasing incident energy. Flyer materials examined include pure aluminum (0.25-2.6 $\mu \mathrm{m}$ thick) and composite targets $(0.5-2.0 \mu \mathrm{m}$ thick) containing an insulating layer of aluminum oxide. While flyer breakup is observed in both types of material, the $\mathrm{Al}_{2} \mathrm{O}_{3}$ barrier significantly delays the deleterious effects of deep thermal diffusion.
\end{abstract}

\section{INTRODUCTION}

Experimental methods for generating laser-driven flyers provide a promising approach to the development of a laboratory-scale capability for quantitative studies of material response to well-controlled, short pulse shock compression. Laser-launched aluminum flyers can easily achieve velocities $>3 \mathrm{~km}-\mathrm{s}^{-1}$. Hence, as impactors, flyers can generate shock pressures well in excess of $10 \mathrm{GPa}$ in many materials. Shock durations, on the other hand, are typically only a few ns or less (1). Properly configured, a laser driver and optical transmission system (typically using multimode, step-index optical tapers and/or fibers to couple the high-intensity optical radiation to the flyer target) can generate a reasonably uniform spatial intensity profile at the target plane, promoting nearly planar launch conditions with precisely variable impact velocities and very accurate $(\sim 1 \mathrm{~ns})$ timing. Accordingly, laser-driven flyers are well suited

\footnotetext{
*This work supported by the U. S. Department of Energy at SNL under contract DE-AC04-94AL85000 and at LLNL under contract W-7405-ENG48.
}

for initiation and probing of shock-induced processes that occur with very short (i.e., nanosecond) characteristic time scales. Laser-driven plates are also potentially useful impactors in the examination (e.g., equation of state studies) of rare, expensive or highly toxic materials.

The dynamic behavior of laser-accelerated flyers is another important factor in determining their utility in shock compression studies. In addition to providing acceptable planarity on impact, it is essential that the flyer arrive at the impact plane either fully intact and at solid density or with dynamic physical properties that can be adequately characterized. Recent high-speed imaging studies (2) have shown that a number of complex, multidimensional effects can occur on a small scale during flyer acceleration. These phenomena include flyer "tilt" (due to a slightly uneven distribution of optical intensity at the fiber output) and fine-structure nonplanarity (arising from modal noise in the intensity distribution). Additional complications are likely to arise from the very high temperatures $(\sim 50000 \mathrm{~K})$ generated in the driving plasma (3). Rapid heat transfer may be expected to result in melting/vaporization
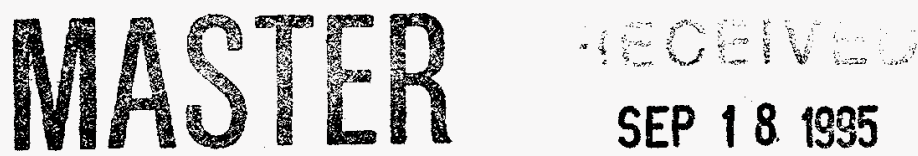


\section{DISCLAIMER}

This report was prepared as an account of work sponsored by an agency of the United States Government. Neither the United States Government nor any agency thereof, nor any of their employees, make any warranty, express or implied, or assumes any legal liability or responsibility for the accuracy, completeness, or usefulness of any information, apparatus, product, or process disclosed, or represents that its use would not infringe privately owned rights. Reference herein to any specific commercial product, process, or service by trade name, trademark, manufacturer, or otherwise does not necessarily constitute or imply its endorsement, recommendation, or favoring by the United States Government or any agency thereof. The views and opinions of authors expressed herein do not necessarily state or reflect those of the United States Government or any agency thereof. 


\section{DISCLAIMER}

Portions of this document may be illegible in electronic image products. Images are produced from the best available original document. 
of some or all of the flyer (depending on the target thickness), quickly leading to a situation where the expanding, relatively low-density plasma pushes against fluid material at higher density. These conditions favor the growth of instabilities. In this paper, we discuss tests in which high-speed streak imaging and simultaneous stop motion microphotography have been used to examine the limiting case of very thin flyers. Results show that rapid melt does occur under these conditions, followed by plasma penetration of the fluid material. The streak imaging technique allows characteristic time scales for phase transformation and plasma breakout to be determined. These effects have been studied as a function of both flyer target thickness and driving energy. In addition, we compare the response of pure aluminum flyers vs. composite materials containing an insulating layer of aluminum oxide.

\section{EXPERIMENTAL}

Flyers were generated using techniques described in detail previously (4). The driving laser employed in this work was a Q-switched Cr:Nd:GSGG laser (Allied Signal) that provided an output $>100 \mathrm{~mJ}$ with a pulse duration near $13 \mathrm{~ns}$. Absorbing neutral density filters were used to vary the energy incident on the flyer targets. These targets were prepared by physical vapor deposition on polished output ends of 400- $\mu \mathrm{m}$-diameter multimode optical fibers. Tests were performed on pure aluminum ranging from $0.25-2.6 \mu \mathrm{m}$ in thickness as well as composite samples spanning a more limited range $(0.5-2.0 \mu \mathrm{m})$.

The principal elements of the optical assembly used for simultaneous streak imaging and stop motion microphotography have also been discussed previously (2). Briefly summarized, the setup utilized a large-format framing camera and a streak camera that viewed the flyer target through a common, fast photographic objective in combination with separate magnifying lens assemblies. Illumination for the framing camera was provided by a shortpulse ( I ns FWHM) broad band dye laser. The optical properties of this illuminator enabled the acquisition of stop-motion images of rapidly accelerating flyers with negligible interference from laser speckle. The dye laser energy was focused to a circular region matching the field of view of the framing

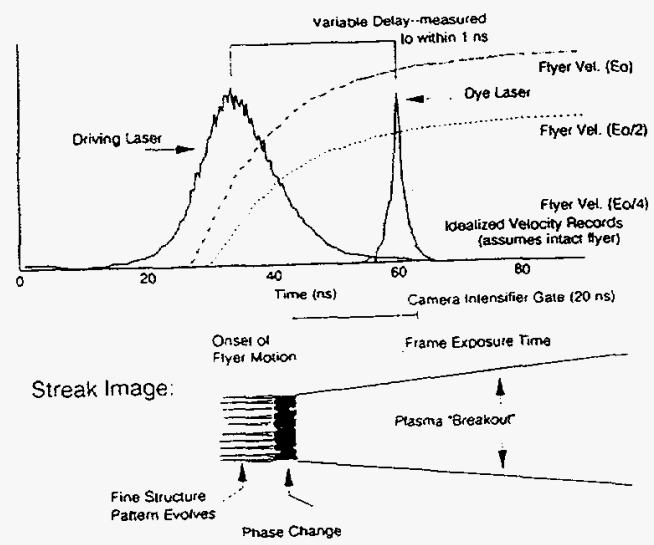

FIGURE 1. Schematic diagram illustrating timing of framing and streak diagnostics relative to onset of flyer motion. At lower driving energies, flyer motion occurs later in the incident pulse

camera. The target surface was viewed at an angle that directed the specular reflection of the illumination beam just outside the aperture of the objective. This orientation yielded a high contrast image in which extremely small angular perturbations on the surface were highly visible. For streak imaging, illumination was supplied by a continuous wave $\mathrm{Ar}^{+}$laser. This source was focused to a line matching the entrance slit of the streak camera. Both streak and framing images were recorded on TMAX-400 film.

Principal elements of the timing sequence for these tests are illustrated in Fig. 1. Digital delay generators were used to set timing for the driving laser, pulsed dye laser, framing camera intensifier gate, etc. Propagation times in the driving laser and dye laser optical transmission systems were carefully measured in order to derive the actual delay between arrival of the two lasers at the target plane from pulse records acquired by on-line beam monitors. In addition, a dye laser fiducial pulse was recorded on the streak camera trace in order to relate the timing of the two diagnostics. The onset of flyer motion relative to the driving laser pulse was not explicitly measured in these experiments; however, a reasonably accurate estimate of the time for this event could be inferred from velocity interferometer measurements in a similar experimental system (5). In this manner, the temporal relationship of all relevant phenomena was established. 


\section{RESULTS AND DISCUSSION}

Figure 2 displays the response of $1-\mu \mathrm{m}$ aluminum targets to two different driving energies as recorded by streak imaging. Important physical effects in these records can be compared to the schematic representation in Fig. 1. Within a few nanoseconds of the onset of motion, a fine pattern of light and dark regions develops in the image of $\mathrm{Ar}^{+}$laser light reflected from the target surface. The spatial frequency in this pattern is consistent with stop-motion photographs of the fine-structure nonplanarity driven by modal noise at the fiber output (2). The pattern steadily develops a coarser structure and then evolves into a condition of very low reflectivity. After another short period, the image becomes dominated by plasma light. The latter effects appear to correspond to melting of the flyer followed by plasma penetration of the fluid material. The onset of a transition to the "dark phase" can be seen in the photograph ("face-on" view) shown in Fig. 3. This picture reveals extended dark regions in the midst of the structure due to dynamic surface roughness (exaggerated by the imaging technique). Other data clearly show fluid flow associated with the "dark phase" $(2)$ and temporal correspondence between localized onset of low reflectivity and plasma appearance (see below).

As illustrated in Fig. 2, the time delay to phase change and plasma "breakout" generally increases (weakly) with decreasing incident energy. The brightness of the emerging plasma is a strong function of energy. Also, higher energy conditions result in obvious venting and radial expansion of the plasma at the perimeter of the accelerating flyer plate. Although the transitions are fairly abrupt, the luminous plasma tends to emanate at discrete points, especially at lower driving energies. Very early appearance of plasma light was observed at one location in the flyer launched at higher energy (Fig. 2a). We attribute this effect to a pinhole in the flyer target. Visual inspection of the films used in these experiments occasionally revealed a small number of these defects, especially with the thinner materials.

In view of the capability of streak imaging to track physical changes arising from rapid heat transfer processes in flyer acceleration, this technique was used to examine the role of a thin, thermally insulating layer of $\mathrm{Al}_{2} \mathrm{O}_{3}$ (deposited in the target material) in

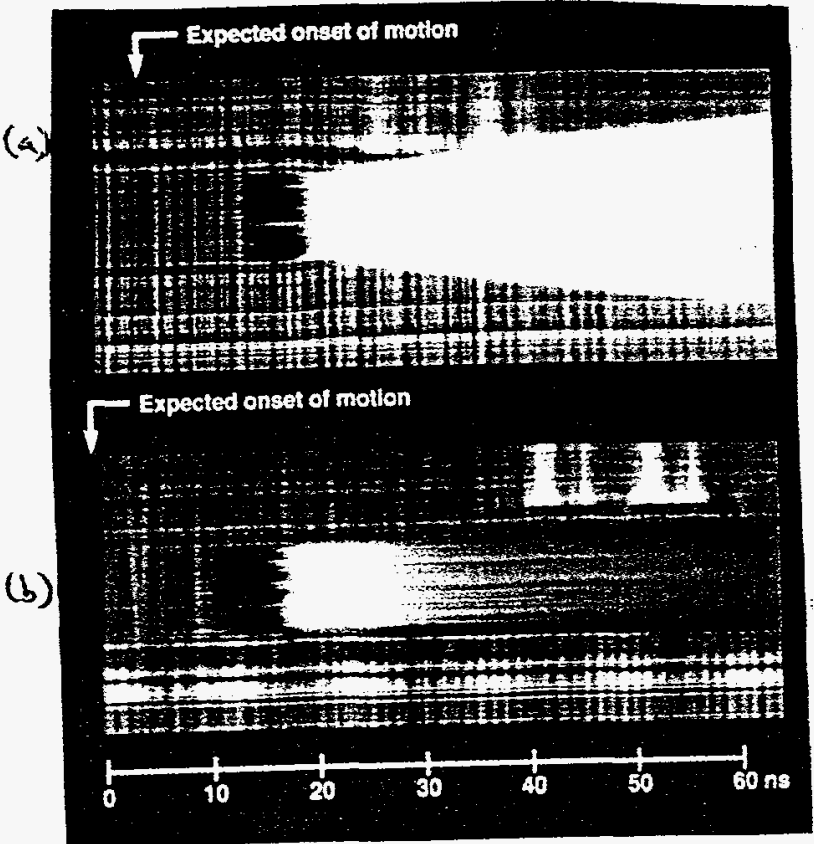

FIGURE 2. Streak records of the response of $1.0-\mu \mathrm{m}$-thick Al films to two different driving energies: (a) $29 \mathrm{~mJ}$; (b) $6.8 \mathrm{~mJ}$. The $400-\mu \mathrm{m}$ diameter of the flyer essentially corresponds to the vertical extent of the dark area.

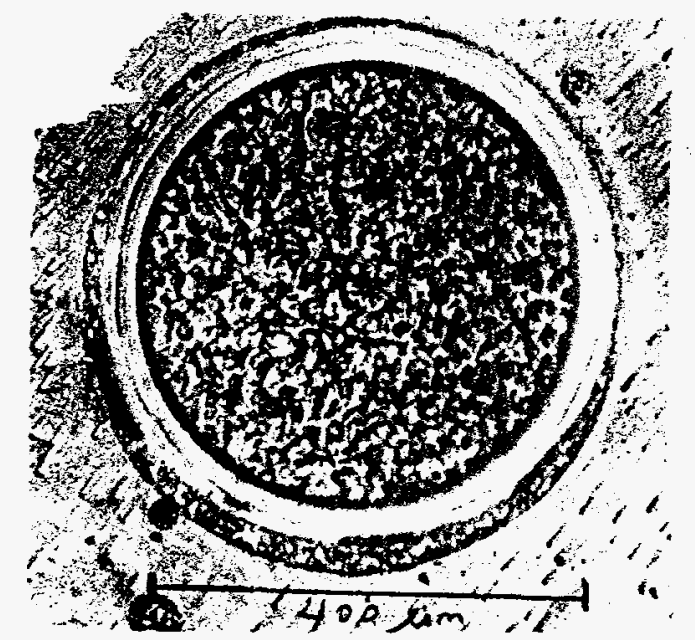

FIGURE 3. Frame image of $2.6-\mu \mathrm{m}$-thick Al flyer, $27.7 \mathrm{~mJ}$ incident energy. Image acquired $-12 \mathrm{~ns}$ after onset of motion.

enhancing flyer performance. It has been shown $(1,6)$ that composite materials of this type yield increased efficiency in converting incident optical energy to flyer kinetic energy, especially near the threshold for flyer motion, and can deliver a shock pulse that shows less evidence of flyer ablation or 
thinning. Figure 4 compares the response of a $2.0-$ $\mu \mathrm{m}$-thick composite film to that of a $2.1-\mu \mathrm{m}$ target of pure Al. Two effects are evident. First, the composite flyer resists melting and plasma penetration for a significantly longer period of time than the aluminum flyer. Second, the transitions are less uniform spatially in the composite case with breakout seemingly occurring at fewer locations across the face of the flyer. Plasma penetration is clearly linked temporally to prior changes in flyer reflectivity, indicative of an early melt in these regions. Similar effects were observed over a wide range of incident energy as well as over the available range of flyer thickness. Defects in the mechanical or thermal properties of the $\mathrm{Al}_{2} \mathrm{O}_{3}$ layer may contribute to this phenomenon. Alternatively, the less uniform transitions may reflect differences in the growth of instabilities in portions of the flyer in contact with the hot, driving plasma.

Figure 5 presents a plot of the time required for plasma breakout over the flyer face vs. initial target thickness. It is clear that a thermally insulating $\mathrm{Al}_{2} \mathrm{O}_{3}$ layer can not prevent melting and plasma penetration of the thin materials examined in this work; however, the barrier does significantly delay the harmful effects of deep thermal diffusion. The increased delay becomes more apparent as thickness increases.

(a)
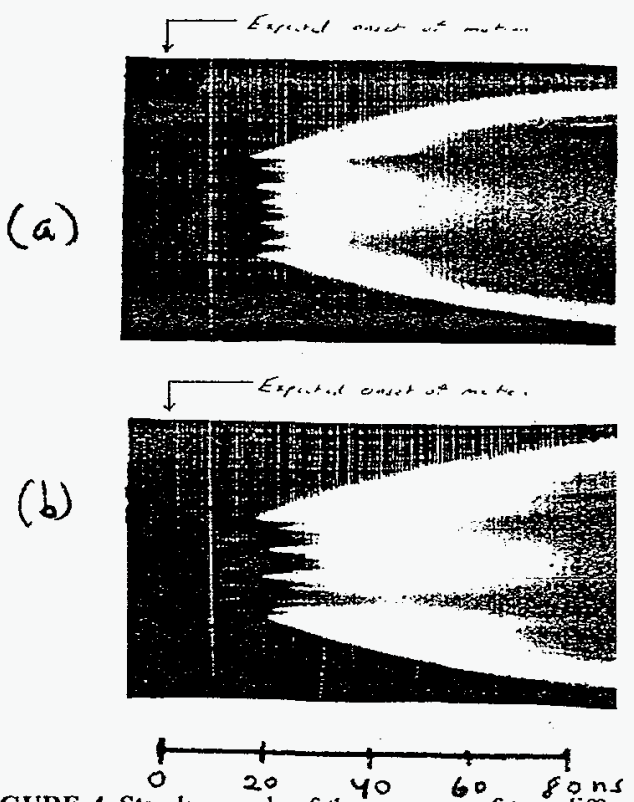

FIGURE 4. Streak records of the response of two different flyer target materials: (a) $2.1-\mu \mathrm{m}$-thick aluminum; (b) 2.0 -mm-thick composite. Incident energy near $28 \mathrm{~mJ}$ in both cases.

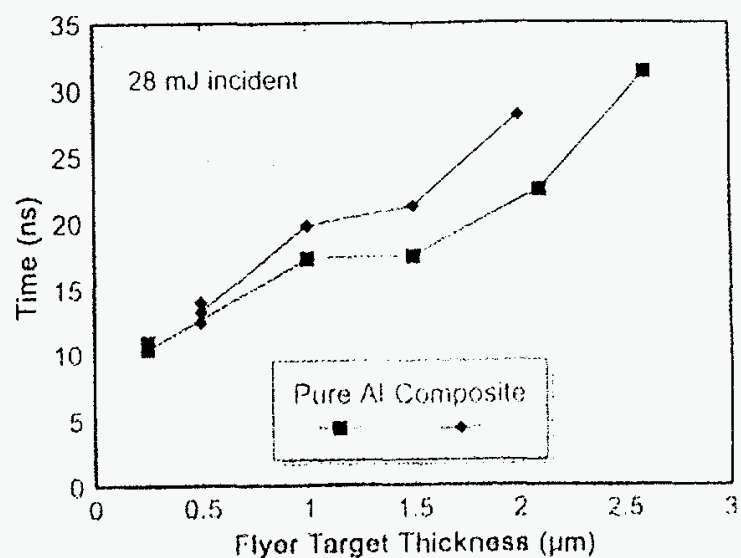

FIGURE 5. Plot of time delay from the onset of flyer motion to the latest plasma breakout across the face of the flyer vs. initial flyer target thickness.

The data presented here highlight some important considerations in the use of laser-driven flyers as impactors for shock compression studies. In principle, high impact velocities can be obtained from laser acceleration of very thin flyers; however, these materials are subject to extremely rapid melting and plasma penetration. Inclusion of a thermally insulating layer in the flyer target can delay but does not prevent flyer breakup. With thicker targets $(>2.5 \mu \mathrm{m})$, a practical "time window" of acceptable flyer integrity can be obtained.

\section{REFERENCES}

1. Trott, W. M., "Investigation of the Dynamic Behavior of Laser-Driven Flyers," in High-Pressure Science and Technology-1993, eds. S. C. Schmidt, J. W. Shaner, G. A. Samara and M. Ross, New York: AIP Press, 1994, pp. 1655 1658.

2. Frank, A. M., and Trott, W. M., "Stop Motion Microphotography of Laser Driven Plates," in Proceedings of Symposium on Ultrahigh- and High-Speed Pholography. Videography and Photonics '94, SPIE Volume 2273, 1994, pp. 196-206.

3. Farnsworth, A. V., "Laser Acceleration of Thin Flyers" (this volume).

4. Trott, W. M., and Meeks, K. D., J. Appl. Phys. 67, 3297-3301 (1990).

5. Trott, W. M., "Studies of Laser-Driven Flyer Acceleration Using Optical Fiber Coupling," in Shock Woves in Condensed Matter--1991, eds. S. C. Schmidt, R. D. Dick, J. W. Forbes, and D. G. Tasker, New York: Elsevier Science Publishers, 1992, pp. 829-832.

6. D. L. Paisley, "Laser-Driven Miniature Flyer Plates for Shock Initiation of Secondary Explosives," in Shock Waves in Condensed Matter--1989, eds. S. C. Schmidh J. N. Johnson, and L. W. Davison, New York: Elsevier Science Publishers, 1990, pp. 733-736. 